\title{
Fault-Tolerant Architecture with Dynamic Wavelength and Bandwidth Allocation Scheme in WDM-EPON
}

\author{
I-Shyan Hwang, Zen-Der Shyu, Chun-Che Chang
}

\begin{abstract}
This study proposes a novel fault-tolerant architecture in WDM-EPON, Cost-based Fault-tolerant WDM-EPON (CFT-WDM-EPON), to provide overall protection. The CFT-WDM-EPON only equips a backup feeder fiber to recover the system failure. Additionally, a prediction-based fair wavelength and bandwidth allocation (PFWBA) scheme is also proposed to enhance the differentiated services for WDM-EPON based on the Dynamic Wavelength Allocation (DWA) and Prediction-based Fair Excessive Bandwidth Reallocation (PFEBR) which is our previous work. The PFEBR involves an Early-DBA mechanism, which improves prediction accuracy by delaying report messages of unstable traffic ONUs, and assigns linear estimation credit to predict the arrival of traffic during waiting time. The DWA can operate in coordination with an unstable degree list to allocate the available time of wavelength precisely. Simulation results show that the proposed PFWBA scheme outperforms the WDM IPACT-ST with a single polling table and the Dynamic wavelength and bandwidth 3 (DWBA3) in terms of end-to-end delay and jitter performance.
\end{abstract}

Index Terms-Fault tolerance, WDM-EPON, differentiated services, PFEBR.

\section{INTRODUCTION}

Compared with the current access network technologies, passive optical network (PON) technologies are a promising solution for the full service access network, since optical fiber can satisfy the increasing bandwidth demand. The ITU-T recommends a series of ATM-based Broadband PON systems (i.e. ATM-PON, BPON and GPON). Furthermore, Ethernet PON (EPON) has been discussed in IEEE 802.3ah as an extension of Gigabit-Ethernet [1]. Although the EPON or ATM-based PON provides higher bandwidth than traditional copper-based access networks, the bandwidth of the PON needs to be increased further. The WDM-PON architecture can adopt wavelength-division multiple access (WDMA) to support multiple wavelengths in either or both upstream and downstream directions [2]. For employing the WDMA technology, the passive arrayed waveguide grating

I-Shyan Hwang is with the Department of Computer Engineering and Science, University of Yuan-Ze, Chung-Li, Taiwan, 32026 (phone: 886-3-4638800; fax: 886-3-4638850; e-mail: ishwang@saturn.yzu.edu.tw).

Zen-Der Shyu is with the Department of Computer Engineering and Science, University of Yuan-Ze, Chung-Li, Taiwan, 32026 (e-mail: s949102@mail.yzu.edu.tw).

Chun-Che Chang is with the Department of Computer Engineering and Science, University of Yuan-Ze, Chung-Li, Taiwan, 32026 (e-mail: s946065@mail.yzu.edu.tw).
(AWG) is deployed in the WDM-PON architecture. The AWG allows for spatial reuse of the wavelength channels; thus, a multi-wavelength source at the OLT is used to transmit multiple wavelengths to the various optical network units (ONUs) [3]. In the WDM-PON, such a framework provides guaranteed Quality-of-Service (QoS), high security and privacy. However, the limitations of WDM-PON are lack of mature device technologies, lack of suitable network protocols and software to support the architecture, and high overall cost of deploying optical modules [4]. To integrate the advantages of EPON and WDM-PON to provide high link capacity, and to lower the overall system cost, a smooth migration to WDMA from EPON is expected a promising solution for optical access network technology.

The WDM-EPON is the expected solution which employs EPON and WDM-PON systems to provide additional link capacity and lower the cost of optical units. The WDM-EPON manages different wavelength channels in order to increase the available bandwidth of the EPON, but not to increase the cost of the system [5], [6]. In the WDM-EPON architecture, the optical line terminal (OLT) node is upgraded as an array of fixed-tuned transceivers, and reserves one control wavelength channel for the OLT to forward broadcast frames to each ONU. For the ONU node structure, the WDM-EPON adds tunable transceivers which employ different tuning times and tuning ranges. In the downstream direction, WDM-EPON broadcasts control messages from the OLT to each ONU through the entire bandwidth of one wavelength. Each ONU discards or accepts the incoming frames depending on the packet header addressing. In the upstream direction, WDM-EPON adopts time-division multiple access (TDMA) coupled with multi-point control protocol data unit (MPCPDU) mechanism to avoid collision. The MPCPDU involves both GATE MPCPDU and REPORT messages. The OLT allocates upstream bandwidth to each ONU by sending GATE MPCPDU messages which contains a timestamp, granted timeslots and wavelengths indicating the periods during which the ONU can transmit data. Each ONU can send REPORT messages concerning the queue state to the OLT, enabling the OLT to allocate the appropriate upstream bandwidth, wavelengths and timeslots to each ONU. With multiple ONUs sharing the same upstream bandwidth and wavelengths to transmit data on the WDM-EPON, any data collision lengthens the end-to-end delay and degrades the system performance. Hence, the bandwidth and wavelength allocation is a major concern of research in the WDM-EPON, 
especially with the large demand for bandwidth and critical applications [5], [6].

Wavelength and bandwidth allocation schemes can be divided into two categories, Static Wavelength Dynamic Time (SWDT) and Dynamic Wavelength Dynamic Time (DWDT) which is also called Dynamic Wavelength and Bandwidth Allocation (DWBA) [7]. In the SWDT, the OLT allocates wavelengths statically and timeslots dynamically. The ONUs are divided into different groups according to the number of wavelengths, and each group of ONUs shares a pre-defined wavelength. However, the number of ONUs on each wavelength is identified in the SWDT, which does not exploit the inter-channel statistical multiplexing, thus lowering utilization. The DWBA assigns the bandwidth and wavelength based on the requested bandwidth, wavelength loading and QoS requirement by each ONU [6], [7]. The DWBA can also exploit both inter-channel and intra-channel statistical multiplexing. Therefore, the DWBA scheme provides more efficient bandwidth allocation than the SWDT scheme, allowing each ONU to share the network resources, and improving QoS for end-users.

Another significant issue in the WDM-EPON is how to protect and recover the optical failure in the WDM-EPON system. Since optical passive networks transmit aggregated high-speed data from several hundreds of end-users, failure in network units or links results in serious data loss. This study proposes a novel fault-tolerant architecture for WDM-EPON, Cost-based Fault-tolerant WDM-EPON $(C F T-W D M-E P O N)$, to lower the cost of conventional protection architecture, and in the short term to recover the functionality of the failed equipment. The CFT-WDM-EPON can recover the optical failure by fast wavelength switching between control and data channel, and only equips a backup feeder fiber to connect the adjacent PON system. Additionally, this study also proposes a robust prediction-based fair wavelength and bandwidth allocation (PFWBA) scheme, which includes the dynamic wavelength allocation (DWA) [7] and Early DBA (E-DBA) mechanism [14]. The E-DBA mechanism for prediction-based fair excessive bandwidth reallocation (PFEBR) scheme is our previous research. The E-DBA reduces the idle period and waiting time in the conventional DBA scheme, and obtains fresh queue information for unstable traffic ONUs to improve the accurate prediction. The DWA can operate in coordination with the unstable degree list to allocate the wavelength available time precisely. Furthermore, to improve the system performance, this study also considers the fairness of excessive bandwidth reallocation among ONUs for differentiated traffic classes.

The rest of this paper is organized as follows. Section 2 describes related work of DWBA and existing protection scheme in WDM-EPON. Section 3 then proposes a novel fault-tolerant architecture, CFT-WDM-EPON, capable of providing overall protection for optical nodes and fibers. Next, Section 4 presents the PFWBA scheme, which incorporates the DWA and the E-DBA mechanism for dealing with prediction and fairness allocation of wavelength and bandwidth. Section 5 presents the simulation results of the proposed system and other well-known methodologies. Conclusions are finally drawn in Section 6.

\section{RELATED WORK}

In WDM-EPON, allocating the bandwidth and wavelength efficiently is the key factor to satisfying various QoS requirements for end-users. Recent studies on wavelength and bandwidth allocation in WDM-EPON can be classified as dynamic wavelength allocation (DWA) and dynamic bandwidth allocation (DBA). The WDM-EPON DWA concerns how the OLT allocates suitable wavelength from multiple wavelengths to ONUs. The WDM-EPON DBA is applied, after the OLT assigns wavelengths to ONUs, in order to allocate bandwidth for each ONU efficiently according to the QoS requirement and network traffic.

Previously proposed DWA systems include the sequential scheduling algorithm [8], which emulates a virtual global first-in-first-out queuing for all incoming requests, and assigns a suitable wavelength for each request. This scheduling algorithm may suffer from wasted bandwidth and poor fairness guarantee if some ONUs have large Round Trip Times (RTTs). To overcome the wasted bandwidth problem, K.S. Kim et al. [9] presents a batch scheduling system that provides priority queuing by scheduling over more than one frame. The batch scheduling system stores the bandwidth requests arriving at OLT during the batch period in queues, and schedules them at the end of batch period. The scheduling delay of the batch scheduling system may increase when the system load is very low and the batch period is short [10]. M. McGarry et al. investigated another scheduling algorithm for REPORT messages such as online scheduling and offline scheduling [5]. In online scheduling, the OLT follows a grant-on-the-fly manner to allocation timeslots. The OLT allocates a transmission window for each ONU as soon as the OLT receives REPORT message from each ONU for the next cycle. Unlike the online scheduling, the OLT follows a wait-for-all manner in offline scheduling. The OLT allocates transmission windows for all ONUs in a round-robin manner after having received all REPORT messages from all ONUs for the next cycle. The offline scheduling with wait-for-all leads to a long waiting time and idle period because of the long inter-scheduling cycle gap.

In terms of WDM-EPON DBA, K.H. Kwong et al. [15] proposed the WDM IPACT-ST scheme based on the interleaved polling with adaptive cycle time (IPACT), which is proposed for EPON access network [1]. The WDM IPACT-ST applies IPACT to multi-channel PON, where ONUs are equipped with fixed transceivers. Nonetheless, the WDM IPACT-ST lacks the ability to handle the excessive bandwidth, which is collected from lightly-loaded ONUs. As an extension of the WDM IPACT-ST, the excessive bandwidth reallocation (EBR) [7], [16] redistributes the available excessive bandwidth to heavily-loaded ONUs according to the proportion of each request, and improves the performance in terms of packet delay. However, EBR has some drawbacks, namely unfairness and excessive bandwidth allocated to ONUs over that requested. This is termed the redundant bandwidth problem [15]. A.R. Dhaini et al. [7] proposed the DWBA3 scheme, the extension of the EBR in the WDM-EPON, which allocates the bandwidth for two steps. The DWBA3 allocates first the guaranteed bandwidth for heavily-loaded ONUs, and the requested bandwidth for lightly-loaded ONUs. Finally, upon receiving 
all REPORT messages, the DWBA3 redistributes the available excessive bandwidth to heavily-loaded ONUs based on the proportion of each request in next cycle. The upstream in different transmission cycle for heavily-loaded ONUs increases the number of guard time, which decreases the available bandwidth, and increases the end-to-end delay.

The PFEBR [14] executes the DBA scheme after the REPORT messages from unstable traffic ONUs are received at the end of $\mathrm{ONU}_{N-1}$, instead of at the end of $\mathrm{ONU}_{N}$ in the standard DBA scheme. The operation reduces the idle period in the standard DBA scheme, and obtains more fresh information of unstable traffic ONUs to enhance the accuracy of prediction in the following cycle. Additionally, the bandwidth is allocated to each ONU in the next cycle according to the unstable degree list. The unstable degree list is calculated using variance of historical traffic, and sorted in decreasing order of all ONUs. The DBA scheme of the PFEBR alleviates traffic variance by shortening the waiting time before transmitting data for unstable traffic ONUs, and thus improves prediction accuracy.

The fault tolerance of WDM-based PON topology has been discussed recently [8]-[13]. The fault-tolerant architecture considers two types of network failure, namely link failure and node failure. The node protection of PON concentrates on the most important optical unit, namely OLT, and the link protection focuses on the feeder fibers that connect the OLT node with the remote node (RN) in the PON system. The protection mechanism for the PON topology constructs the backup links or nodes to recover the failure. The authors of [8], [13] provide recovery mechanism against fiber-cut of feeder fiber which connects RN and OLT. H. Nakamura et al. [13] presented the protection fiber for feeder fibers to avoid the fiber-cut situation. The protection fiber may recover fiber failure occurring on the working fiber, but cannot recover from failure occurring on OLT. F.T. An et al. [8] proposed a new Hybrid TDM/WDM-PON architecture named Stanford University aCCESS Hybrid WDM/TDM Passive Optical Network (SUCCESS-HPON). The SUCCESS-HPON is based on a ring-plus-distribution-trees topology, which can provide users with better protection and restoration capabilities than conventional PONs. The SUCCESS provides bi-directional transmission on the same wavelength and fiber. Transmission occurs in reverse direction to recover the failure if the feeder fiber fails. However, the SUCCESS-HPON cannot provide protection for OLT and ONUs. Furthermore, the ring-plus-distribution-trees topology has a long round trip time (RTT) due to the very large number of ONUs. The authors of [11], [12] proposed redundant fibers topology to avoid the failure occurs. However, the protection for feeder and distribution fiber is provided by duplicate deployment of fiber in [11], which is not a cost-effective protection scheme. Furthermore, this protection architecture does not provide any recovery system for node failure. X. F. Sun et al. presented a ring-plus-tree architecture, which not only constructs protection links for feeder fibers, but also constructs protection links between ONUs [12]. The ONU still can transmit data by protection fiber connecting neighboring ONUs when the fiber-cut occurs on the distribution fibers. However, the protection architecture still cannot prevent node failure on optical nodes, and is not cost-effective due to duplicating deployment of fibers. The above models focus on the fiber-cut situation on optical fibers, but do not consider optical node failures, even if the node failure may damage the PON system. If optical node failure occurs on the OLT, then the dynamic wavelength and bandwidth allocation cannot be processed. Additionally, the bandwidth and wavelength requested by all ONUs cannot be granted without leading to significant damage. Therefore, a good fault-tolerant scheme should protect the most important optical nodes, the OLT and the feeder fiber to prevent the failures that degrade the system performance.

\section{The Proposed CFT-WDM-EPON ARCHITECTURE}

The proposed protection architecture, CFT-WDM-EPON, based on WDM-EPON, comprises a centralized optical line terminal (OLT) and passive arrayed waveguide grating (AWG), and connects a group of associated optical network units (ONUs) to provide complete protection for overall PON architecture, illustrated in Fig. 1. Each PON system connects adjacent PON systems by protection feeder fiber, and each AWG connects two OLTs with many ONUs. When no failures occur on the CFT-WDM-EPON, ONUs transmit the REPORT message to OLTs through wavelength, $\lambda_{\text {REPORT }}$, which is reserved for transmitting control messages for each ONU. The OLT considers takes the loading balancing between different wavelengths when the OLT assigns wavelengths and bandwidth to each ONU by GATE MPCPDU message. The ONUs transmit data by different wavelengths, $\lambda_{\alpha}$ and $\lambda_{\beta}$, which are routed and transmitted through different feeder fibers by AWG. When the failure occurs on the CFT-WDM-EPON, OLT \#1 cannot receive the REPORT messages from the ONUs in PON \#1, and OLT \#2 will starts the recovery scheme to take over the REPORT message from PON $\# 1$, and allocate wavelengths, $\lambda_{\text {REPORT }}$ and $\lambda_{\beta}$, for upstream through protection feeder fiber to PON $\# 1$. The protection architectures in papers [8], [11]-[13] do not provide any recovery scheme for the OLT node, shown in Table 1. To prevent optical node failure and fiber failure from causing significant damage to the PON system, the CFT-WDM-EPON only equips backup optical fiber to provide the overall protection scheme.

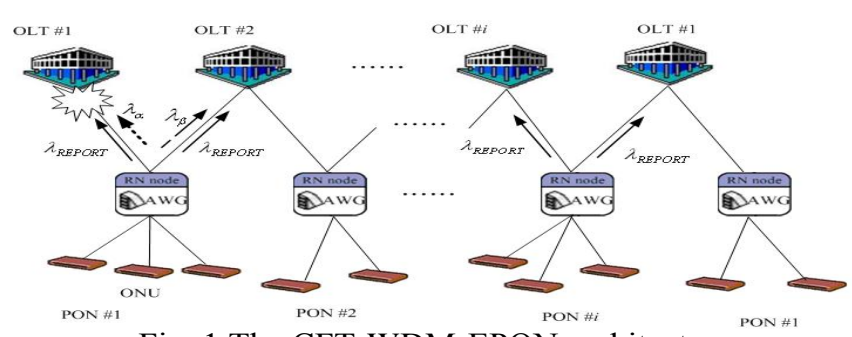

Fig. 1 The CFT-WDM-EPON architecture

Table 1 The Comparisons of the Protection Architecture

\begin{tabular}{|c|c|c|c|c|c|}
\hline Architecture & $\begin{array}{c}\text { CFT- } \\
\text { WDM- } \\
\text { EPON }\end{array}$ & $\begin{array}{c}\text { F.T.An } \\
\text { Eeature }[8]\end{array}$ & $\begin{array}{c}\text { H.Nakamura } \\
\text { et al. }[13]\end{array}$ & $\begin{array}{c}\text { E.S.Son } \\
\text { et al. [11] }\end{array}$ & $\begin{array}{c}\text { X.F.Sun } \\
\text { et al. }[12]\end{array}$ \\
\hline $\begin{array}{c}\text { Erotected OLT } \\
\text { Protected }\end{array}$ & Yes & No & No & No & No \\
\hline Feeder Fiber & Yes & Yes & Yes & Yes & Yes \\
\hline \# PON & $\boldsymbol{N}$ & $\boldsymbol{N}$ & $\boldsymbol{N}$ & $\boldsymbol{N}$ & $\boldsymbol{N}$ \\
\hline \# OLT & $\boldsymbol{N}$ & $\mathbf{1}$ & $\mathbf{1}$ & $\boldsymbol{N}$ & $\boldsymbol{N}$ \\
\hline \# Feeder Fiber & $\mathbf{2 N}$ & $\boldsymbol{N + 1}$ & $\mathbf{2 N}$ & $\mathbf{2 N}$ & $\mathbf{2 N}$ \\
\hline
\end{tabular}




\section{The Proposed Dynamic Wavelength AND BANDWIDTH ALLOCATION}

This study proposes a robust prediction-based fair wavelength and bandwidth allocation (PFWBA) scheme, which includes the dynamic wavelength allocation (DWA) and E-DBA mechanism of the PFEBR scheme. The E-DBA mechanism allocates the bandwidth to each ONU according to the decreasing order of unstable degree list and improves the prediction accuracy. Additionally, the DWA mechanism selects the wavelength with the least available time for each ONU to reduce the average delay time. To reduce the prediction inaccuracy resulting from a long waiting time, the DWA divides all ONUs into three groups based on the unstable degree list. The DWA can cooperate with the PFWBA scheme to select a suitable wavelength, and reduce the delay time for each ONU.

\section{A. PFEBR Scheme with Early DBA Mechanism}

\section{1) The Operation of Early DBA Mechanism}

The E-DBA mechanism arranges the sequence of transmitting REPORT messages to OLT by delaying some unstable traffic ONUs of $\beta_{V}$. The E-DBA mechanism consists of two operations. First, the OLT executes the DBA scheme after the REPORT messages from $\beta_{V}$ are received at the end of $\mathrm{ONU}_{N-1}$, as illustrated in Fig. 2(b), instead of $\mathrm{ONU}_{N}$ in the standard DBA scheme, illustrated in Fig. 2(a). The operation reduces the idle period in the standard DBA scheme, and obtains the fresh queue information for unstable traffic ONUs to improve the prediction accuracy. Second, the bandwidth request for each ONU is allocated based on the traffic variation of all ONUs in decreasing order, and $\beta_{V}$ is updated by assigning some unstable traffic ONUs with higher variations. This operation alleviates variance by shortening the waiting time for unstable traffic ONUs, illustrated in Fig. 2(c), to enhance the prediction accuracy.

\section{2) PFEBR Scheme}

\section{- Unstable degree list}

The PFEBR calculates the variance of each ONU from the historical traffic required, and sorts the variances in decreasing order to obtain the unstable degree list. The variance of $\mathrm{ONU}_{i}, V_{i}$, can be expressed as follows:

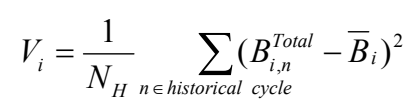

, where $B_{i, n}^{\text {Total }}$ represents the sum of differentiated traffic classes of $\mathrm{ONU}_{i}$ in the $\mathrm{n} t h$ cycle, $\bar{B}_{i}$ is the mean of the $B_{i, n}^{\text {Total }}$, i.e. $\bar{B}_{i}=\frac{1}{N_{H}} \sum_{n=1}^{N_{H}} B_{i, n}^{\text {Total }}$, and $N_{H}$ represents the number of historical REPORT messages piggybacked. $\beta_{V}$ denotes a set of ONUs in unstable degree list with a high variance which is greater than the mean variance $\bar{V}$, where $\bar{V}=\frac{1}{N} \sum_{i=1}^{N} V_{i}$. The bandwidth prediction of each ONU after obtaining the unstable degree list is described as follows. Unlike the mechanism that piggybacks all REPORT messages in the data timeslots, the E-DBA mechanism shifts the REPORT messages of $\beta_{V}$ between the $(N-1)$ th and $\mathrm{N} t h$ ONU. The PFEBR requires the fresh queue information of unstable

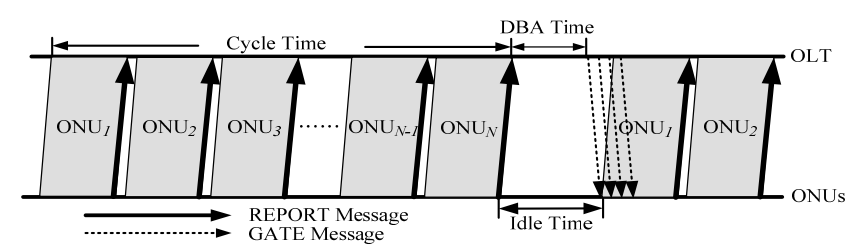

Fig. 2(a) Operation of standard DBA scheme

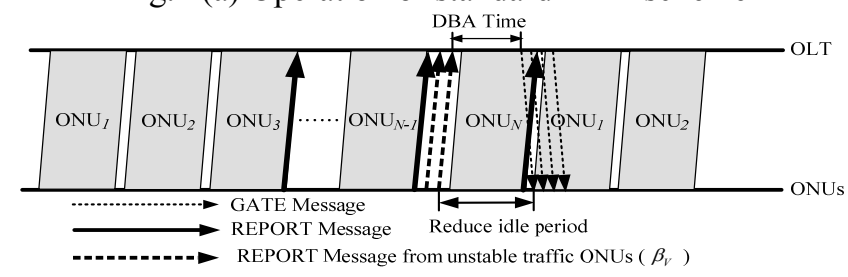

Fig. 2(b) Operation with the proposed E-DBA mechanism

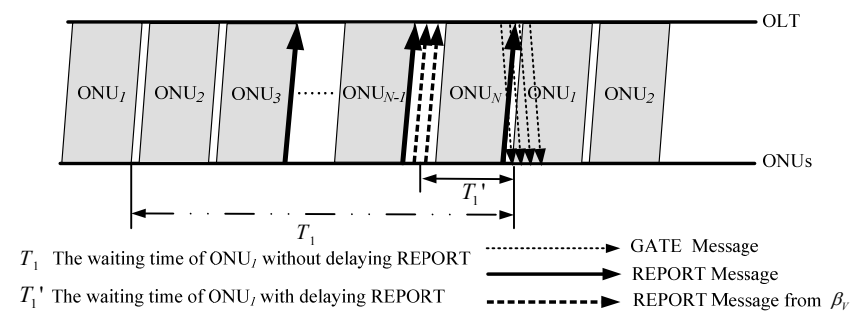

Fig. 2(c) Enhancing prediction accuracy by shortening the waiting time of unstable traffic ONUs

traffic ONUs to avoid prediction inaccuracy, which degrades the system performance.

\section{- Prediction based on unstable degree list}

After the sequence of all ONUs from the unstable degree list is uploaded, the PFEBR predicts the traffic bandwidth required according to the unstable degree list. The predicted request, $R_{i, n+1}^{c}$, for differentiated traffic classes of all ONUs is defined as follows:

$$
\left\{\begin{array}{l}
R_{i, n}^{E F}=B_{i, n}^{E F} \\
R_{i, n+1}^{c}=(1+\alpha) B_{i, n}^{c}, c \in\{A F, B E\}
\end{array}\right.
$$

, where $B_{i, n}^{c}$ represents the requested bandwidth of $\mathrm{ONU}_{i}$ in the $\mathrm{n}$ th cycle, for differentiated traffic classes $c \in\{A F, B E\}$, and $\alpha$ denotes the linear estimation credit modified from the PFEBR [14]. To achieve a better performance for a time-critical application, such as EF traffic, the constant bit rate (CBR) bandwidth should be assigned to the ONUs according to the rate of these applications. Therefore, this study assigns the CBR bandwidth to EF traffic.

\section{- Excessive bandwidth allocation}

The PFEBR executes the EBR to assign uplink bandwidth to each ONU after it has finished predicting the bandwidth needed for each ONU. The proposed PFEBR scheme can provide fairness for excessive bandwidth allocation according to the guaranteed bandwidth rather than requested bandwidth [16], with no partiality or increase in bandwidth utilization. The operation of fair EBR in the PFEBR is described as follows. First, calculate the $R_{i, n}^{\text {Total }}$ of all ONUs. The available bandwidth, $B_{\text {available }}$, can be expressed as

$$
B_{\text {available }}=C_{\text {capacity }} \times\left(T_{\text {cycle }}-N \cdot g-N_{v} \cdot g\right)-N \times 512
$$

, where $C_{\text {capacity }}$ represents the OLT link capacity (bits/sec), $T_{\text {cycle }}$ denotes the maximum cycle time; $g$ is the guard time; $N$ is the number of ONUs, and $N_{V}$ is the number of ONUs 
in $\beta_{V}$. The $\mathrm{ONU}_{i}$ with the maximal residue bandwidth, i.e. $\max \left(S_{i}-R_{i, n}^{\text {Total }}\right)$, is then selected from unassigned ONUs. The granted bandwidth for $\mathrm{ONU}_{i}, G_{i, n+1}^{\text {Total }}$, is given as follows:

$$
G_{i, n+1}^{\text {Total }}=\min \left(B_{\text {available }} \times \frac{S_{i}}{\sum_{k \in \text { unassigned }} S_{k}}, R_{i, n}^{\text {Total }}\right)
$$

, where $R_{i, n}^{\text {Total }}$ represents the sum of the differentiated traffic load after being predicted from $\mathrm{ONU}_{i}$ in the nth cycle; $S_{i} \cdot\left(\sum_{k \in \text { unassigned }} S_{k}\right)^{-1}$ is the proportion of available bandwidth, $B_{\text {available }}$, granted to $\mathrm{ONU}_{i}$. The granted bandwidth for $\mathrm{EF}, \mathrm{AF}$ and $\mathrm{BE}$ classes are described as follows:

$$
\left\{\begin{array}{l}
G_{i, n+1}^{E F}=R_{i, n}^{E F} \\
G_{i, n+1}^{A F}=\min \left(G_{i, n+1}^{\text {Total }}-G_{i, n+1}^{E F}, R_{i, n}^{A F}\right) . \\
G_{i, n+1}^{B E}=G_{i, n+1}^{\text {Total }}-G_{i, n+1}^{E F}-G_{i, n+1}^{A F}
\end{array}\right.
$$

The process $B_{\text {available }}=B_{\text {available }}-G_{i, n+1}^{\text {Total }}$ continues until all ONUs has been assigned. Finally, the PFEBR arranges the upload sequence of each ONU by unstable degree list.

\section{B. Dynamic Wavelength Allocation}

The PFWBA defines the following global status variables used in the scheme description:

1) CAT: Channel available times. $C A T[i]=t$ indicates that the wavelength $\lambda_{i}$ is available for transmission after time $t$, where $i=1,2, \ldots, w$, and $w$ is the number of wavelengths.

2) RTT: RTT[i] represents the round trip time (RTTs) between the OLT and the ith $O N U$.

The PFWBA considers the unstable degree list when scheduling the upload sequence after collecting all REPORT messages from the ONUs in order to improve the prediction accuracy. First, the PFWBA divides all ONUs into three levels based on the variance of all ONUs and allocates the wavelength for each ONU group by group, which is determined as follows:

$$
\left\{\begin{array}{ll}
\text { Group 1, } & \text { if } \mathrm{ONU}_{i} \in \beta_{V} \\
\text { Group 2, } & V_{i}>\bar{V} \text { and } \mathrm{ONU}_{i} \notin \beta_{V} \\
\text { Group 3, } & \text { otherwise }
\end{array} .\right.
$$

The wavelength scheduling process is described as follows.

1) Schedule the PFWBA from Group1 to Group3 according to the unstable degree list.

2) Select the requested frame in the same Group with the minimum transmission time, and schedule its transmission.

Transmission time $=R T T[i]+g+$ transmission timeslots , where $g$ is the guard time, and the transmission timeslots are obtained from the PFEBR.

3) Choose the earliest available wavelength transmission time $C A T[i]$.

4) Update the $C A T[i]$ is as $C A T[i]=$ transmission time + $C A T[i]$.

5) Repeat the above operation until the requested frames in the same Group are scheduled, and schedule the requested frames in the following Group.

\section{V.PERFORMANCE ANALYSIS}

The performance of the proposed PFWBA was compared with that of the WDM IPACT-ST [15] and DWBA3 [7] in terms of end-to-end delay and jitter performance. The performance evaluation was examined by the OPNET simulation tool. Two wavelength channels were adopted, and the link capacity was $1 \mathrm{~Gb} / \mathrm{s}$. The distance from one ONU to the OLT was assumed to be $10-20 \mathrm{~km}$, and each ONU had an infinite buffer. The service policy was first-in first-out. For the traffic model considered here, an extensive study shows that most network traffic can be characterized according to self-similarity and long-range dependence (LRD) [17]. This model was adopted to generate highly bursty $\mathrm{BE}$ and $\mathrm{AF}$ traffic classes with the Hurst parameter of 0.7 . The packet sizes were uniformly distributed between 64 and 1518 bytes. Additionally, high-priority traffic (e.g. voice applications) was modeled by a Poisson distribution, and the packet size was fixed to 70 bytes [18]. The traffic profile was as follows: $20 \%$ of the total generated traffic was considered as high priority, and the remaining $80 \%$ was equally distributed between low- and medium-priority traffic [19].

\section{A. End-to-end delay}

Fig. 3 compares the average end-to-end packet delay of the PFWBA for EF, AF and BE traffics classes with different numbers of wavelengths and ONUs vs. traffic loads. The EF traffics class in the PFWBA with two wavelengths and 64 ONUs had the longest packet delay when the traffic load exceeded 90\%, as shown in Fig. 3(b). The possible reason is that the wavelength scheduling mechanism initially chose the wavelength with the first available time for transmitting the ONUs. The prior selection of wavelength for transmission may result in prediction inaccuracy. The ITU-T recommendation G. 114 specifies the delay for voice traffic in access network at $1.5 \mathrm{~ms}$ [20]. Although the PFWBA scheme with two wavelengths and 64 ONUs had the longest delay, the EF end-to-end delay was still less than $1.5 \mathrm{~ms}$. Fig. 4 compares the end-to-end packet delays among the PFWBA, WDM IPACT-ST and DWBA3 of all EF, AF and BE traffics classes with two channels and 64 ONUs for different traffic loads. Simulation results show that the proposed PFWBA outperformed the other two schemes.

\section{B. EF Jitter Performance}

Fig. 5(a) compares the jitter performance of the PFWBA for EF traffic with different numbers of wavelengths and ONUs vs. traffic loads, respectively. The delay variance $\sigma^{2}$ is calculated as $\sigma^{2}=\frac{\sum_{i=1}^{N}\left(d_{i}^{E F}-\bar{d}\right)^{2}}{N}$, where the $d_{i}^{E F}$ represents the EF delay time of packet $i$, and $N$ is the total number of received EF packets. Simulation results show that the delay variance for EF traffic lengthened when the traffic load rises. However, the PFWBA with two wavelengths and 64 ONUs had the highest delay variance when the traffic load exceeded $90 \%$. The reason is that the number of ONUs with higher variance increased when the traffic load exceeded $90 \%$, causing the unstable degree list to change continuously. Therefore, the wavelength scheduling mechanism and the upload sequence of ONUs also changed continuously. Fig. 


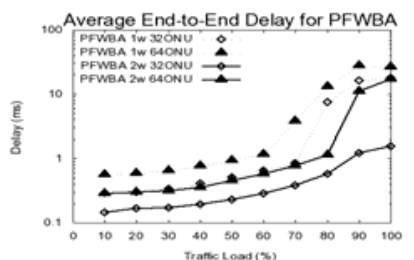

(a)

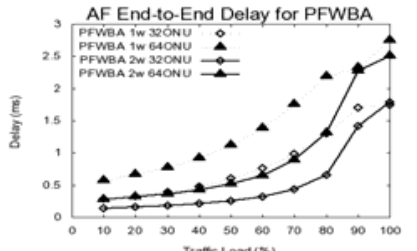

(c)

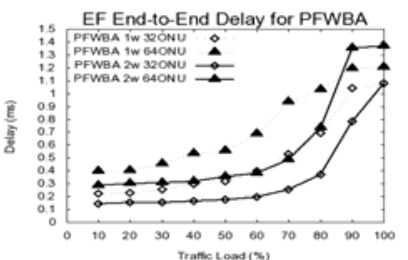

(b)

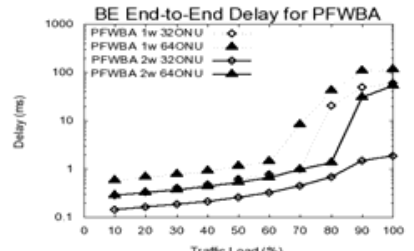

(d)
Fig. 3 End-to-end delay for PFWBA

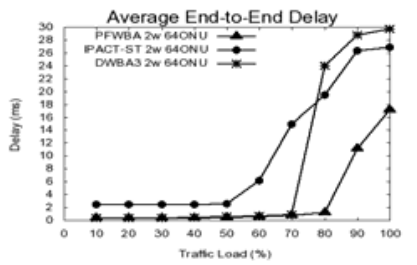

(a)

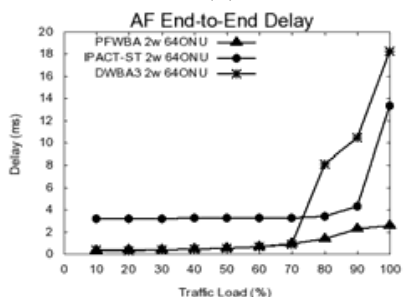

(c)

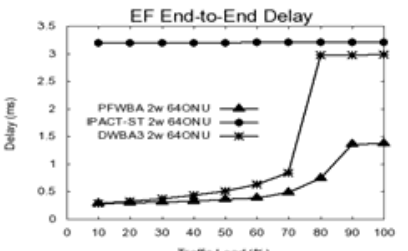

(b)

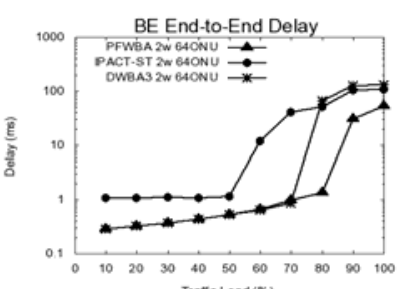

(d)
Fig. 4 End-to-end delay Comparison

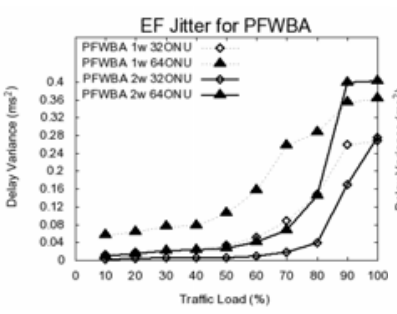

(a) Jitter for PFWBA

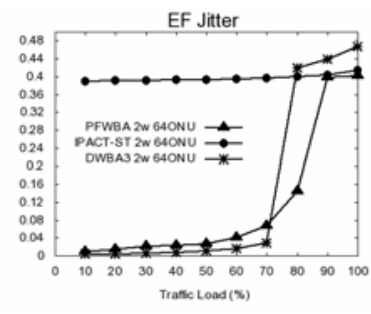

(b) Jitter Comparison
Fig. 5 Delay Variance Comparison

5(b) compares the jitter performance of EF traffic among the PFWBA, WDM IPACT-ST and DWBA3 with two channels and 64 ONUs for different traffic loads. Simulation results show that the proposed PFWBA outperformed the other two schemes for the EF traffic class.

\section{CONCLUSION}

The proposed protection architecture, CFT-WDM-EPON, enables feeder fibers to provide a recovery mechanism. If no failures occur in the PON system, then the CFT-WDM-EPON can share the loading of the working feeder fibers. When the failures occur on the OLT or feeder fibers, the backup fibers will recover the failed ones. Additionally, the PFWBA scheme integrates an efficient dynamic wavelength allocation and E-DBA mechanism of the PFEBR to improve the prediction accuracy and system performance. Simulation results show that the PFWBA can reduce the overall end-to-end delay in differentiated traffic.

\section{REFERENCES}

[1] IEEE 802.3ah task force home page. Available: http://www.ieee802.org/3/efm.

[2] N.J. Frigo, P.P. Iannone, P.D. Magill, T.E. Darcie, M.M. Downs, B.N Desai, U. Koren, T.L. Koch, C. Dragone, H.M. Presby, and G.E. Bodeep, "Wavelength-division multiplexed passive optical network with cost-shared components," IEEE Photonics Technology Letters, vol. 6, issue 11, pp. 1365-1367, Nov. 1994.

[3] S.J. Park, C.H. Lee, K.T. Jeong, H.J. Park, J.G. Ahn, and K.H. Song, "Fiber-to-the-home services based on wavelength division multiplexing passive optical network," Journal of Lightwave Technology, vol. 22, issue 11, pp. 2582-2591, Nov. 2004.

[4] A. Banerjee, Y. Park, F. Clarke, H. Song, S. Yang, G. Kramer, K. Kim, and B. Mukherjee, "Wavelength-division-multiplexed passive optical network (WDM-PON) technologies for broadband access: a review [Invited]", Journal of Optical Networking, vol. 4, no. 11, pp. 737-758, Nov. 2005.

[5] M. McGarry, M. Maier, and M. Reisslein, "WDM Ethernet passive optical networks," IEEE Communications Magazine, vol. 44, issue 2, pp. 15-22, Feb. 2006.

[6] M. McGarry, M. Maier, and M. Reisslein, "An evolutionary WDM upgrade for EPONs," Technical Report (Arizona State University), 2005.

[7] A.R. Dhaini, C.M. Assi, M. Maier, and A. Shami, "Dynamic Wavelength and Bandwidth Allocation in Hybrid TDM/WDM-EPON Networks," Journal of Lightwave Technology, vol. 25, issue 1, pp. 277-286, Jan. 2007.

[8] F.T. An, K.S. Kim, D. Gutierrez, S. Yam, E. (S.T.) Hu, K. Shrikhande, and L.G. Kazovsky, "SUCCESS: A next-generation hybrid WDM/TDM optical access network architecture," Journal of Lightwave Technology, vol. 22, issue 11, pp. 2557-2569, Nov. 2004.

[9] K.S. Kim, D. Gutierrez, F.T. An, and L.G. Kazovsky, "Batch scheduling algorithm for SUCCESS WDM-PON," GLOBECOM IEEE Global Telecommunications Conference, vol. 3, pp. 1835-1839, 2004.

[10] K.S. Kim, D. Gutierrez, F.T. An, and L.G. Kazovsky, "Design and performance analysis of scheduling algorithms for WDM-PON under SUCCESS-HPON architecture," Journal of Lightwave Technology, vol.23, issue 11, pp. 3716-3731, Nov. 2005.

[11] E.S. Son, K.H. Han, J.H. Lee, and Y.C. Chung, "Survivable network architectures for wavelength-division-multiplexed passive optical networks," Photonic Network Communications, vol. 12, issue 1, pp. 111-115, July 2006.

[12] X.F. Sun, Z.X. Wang, C.K. Chan, and L.K. Chen, "A novel star-ring protection architecture scheme for WDM passive optical access networks", Conference on Optical Fiber Communication, Technical Digest Series, vol. 3, Article number 1501381, pp. 563-565, 2005.

[13] H. Nakamura, H. Suzuki, J.I. Kani, and K. Iwatsuki, "Reliable wide-area wavelength division multiplexing passive optical network accommodating gigabit ethernet and 10-Gb ethernet services , Journal of Lightwave Technology, vol. 24, issue 5, pp. 2045-2051, May 2006.

[14] I.S. Hwang, Z.D. Shyu, L.Y. Ke, and C.C. Chang, "A Novel Early DBA Mechanism with Prediction-based Fair Excessive Bandwidth Reallocation Scheme in EPON", The Sixth International Conference on Networking (ICN 2007), Sainte-Luce, Martinique, April 22-28, 2007.

[15] K.H. Kwong, D. Harle, and I. Andonovic, "Dynamic bandwidth allocation algorithm for differentiated services over WDM-EPONS," $9^{\text {th }}$ IEEE Singapore International Conference on Communication Systems, pp. 116-120, 2004.

[16] J. Zheng, "Efficient bandwidth allocation algorithm for Ethernet passive optical networks," IEE Proceedings Communications, vol. 153, issue 3, pp. 464-468, June 2006.

[17] W. Willinger, M.S. Taqqu, and A. Erramilli, "A bibliographical guide to self-similar traffic and performance modeling for modern high-speed networks," in Stochastic Networks, 1996. F. P. Kelly, S. Zachary and I. Ziedins (eds.), Oxford University Press, Oxford, pp. 339-366, 1996.

[18] S. Blake, D. Black, M. Carlson, E. Davies, Z. Wang, and W. Weiss, “An Architecture for Differentiated Services," IETF RFC 2475, Dec. 1998.

[19] X. Bai and A. Shami, "Modeling Self-Similar Traffic for Network Simulation," Technical report, NetRep-2005-01, April 2005.

[20] ITU-T Recommendation G.114, "One-way transmission time," May 2003. 